\title{
Medical Students Cultural Attitudes: The Health Belief Attitudes Survey
}

\author{
James Earl Corley ${ }^{1}$, F. Stanford Massie Jr. ${ }^{1,2}$, Martha Medrano ${ }^{3}$, Carlos A. Estrada ${ }^{1,2}$ \\ ${ }^{1}$ The University of Alabama School of Medicine, Birmingham, Alabama, USA \\ ${ }^{2}$ Birmingham Veterans Affairs Medical Center, Birmingham, Alabama, USA \\ ${ }^{3}$ University of Texas Health Science Center at San Antonio, San Antonio, Texas, USA \\ Correspondence: Carlos Estrada, MD, MS, The University of Alabama at Birmingham, 720 Faculty Office Tower, 510 \\ 20th Street South, Birmingham, AL 35294-3407, USA.
}

Received: October 19, 2015 Accepted: November 5, 2015 Online Published: November 16, 2015

doi:10.11114/jets.v4i2.1201

URL: http://dx.doi.org/10.11114/jets.v4i2.1201

\begin{abstract}
Cultural competent care is the ability to deliver effective medical care to people from different cultures. The lack of methodological rigor and paucity of psychometric properties information of the instruments limits the generalizability of cultural competency educational interventions. We examined cultural attitudes of first year medical students and examined psychometric properties of the scale to better define the constructs it intends to measure. In a cross-sectional study, first year medical students completed the Health Belief Attitudes Survey (HBAS) in September of their matriculating year (2011-2013) within the context of Introduction to Clinical Medicine. The survey has15 items scored on a 6-point Likert scale (1-6), higher score indicates higher culturally competent attitudes. We used factor analysis to explore constructs and examine internal consistency (Cronbach's alpha). The response rate was 98\% (536/548), 42.2\% students were female $(\mathrm{n}=231), 73.0 \%(\mathrm{n}=400)$ white, $14.6 \%$ Asian $(\mathrm{n}=80)$, and $4.4 \%$ African American $(\mathrm{n}=24)(4.9 \%$, $\mathrm{n}=27$, did not provide race or ethnicity). The HBAS median score was $5.3\left(25^{\text {th }}\right.$ percentile [Q1], $4.9 ; 75^{\text {th }}$ percentile [Q3], 5.7). A two-factor solution explained $97 \%$ of the variance with Eigenvalues of 5.6 and 1.2, respectively. We conceptualized the constructs as "Understanding the Patients' Cultural and Socio-Economic Background" (Factor 1, 11 items; Cronbach's alpha, 0.89). "Building the Professional Relationship and Quality of Care" (Factor 2, 4 items; Cronbach's alpha, 0.74). First year medical students have high culturally-relevant attitudes. The HBAS instrument captures two main constructs, understanding the patients' background and perspective and building the professional relationship.
\end{abstract}

Keywords: cultural competency/education, attitude of health personnel, cultural diversity, psychometrics, reproducibility of results

\section{Introduction}

\subsection{Cultural Competence and Medical Education}

Cultural competent care is the ability to deliver effective medical care to people from different cultures (Betancourt \& Green, 2010). As such, the patient-doctor communication requires understanding, respecting, and negotiating patients' understanding of their illness (Carrillo, Green, \& Betancourt, 1999). Evidence suggests that cultural competency training improves knowledge, attitudes, and clinical outcomes in some, but not all studies (Beach et al., 2005; Horvat, Horey, Romios, \& Kis-Rigo, 2014; Lie, Lee-Rey, Gomez, Bereknyei, \& Braddock, 2011; Truong, Paradies, \& Priest, 2014). However, studies examining interventions lacked methodological rigor (Price et al., 2005); an essential component being the use of assessment tools to assess knowledge, attitudes, and other outcomes.

The Liaison Committee on Medical Education (LCME, 2015) with regards to functions and structure of a medical school requires cultural competency training in all medical schools in the United States. An effective cultural competency education would allow students to avoid stereotyping and empathize with the cultural norms of patients. However, cultural competency training is particularly difficult to teach because students tend to perceive diversity as something that creates problems for healthcare professionals due to patients' perceived differences (Nazar, Kendall, Day, \& Nazar, 2014). Inventories and tools are available to assess cultural competency curricula in medical school curricula. For example, Peña Dolhun et al (Pena Dolhun, Munoz, \& Grumbach, 2003) found considerable variation in approaches of teaching methods, skills examined, and content areas in cross-cultural education among the 19 medical schools that 
supplied curricular materials. Limited in the literature are validated methods to assess cultural competence among medical students (Carter et al., 2006; Ladson, Lin, Flores, \& Magrane, 2006).

\subsection{Assessment Tools}

A systematic review found that most studies that used surveys and other assessment tools for reporting attitudes have not been validated (Beach et al., 2005); of the 45 self-administered instruments identified, 22 were unavailable for review and only six had reliability and validity reported. The lack of methodological rigor and paucity of psychometric properties information of the instruments limits the generalizability of cultural competency educational interventions. Efforts continue to rigorously examine the psychometric properties of instruments to assess cultural competency among health professionals (Harris-Haywood et al., 2014; Kardong-Edgren \& Campinha-Bacote, 2008; Loftin, Hartin, Branson, \& Reyes, 2013).

The Health Beliefs and Attitudes Scale (HBAS) was developed to determine students' cultural competency attitudes during the medical interview (Crosson, Deng, Brazeau, Boyd, \& Soto-Greene, 2004; Dobbie, Medrano, Tysinger, \& Olney, 2003). The scale showed moderate internal consistency (Cronbach's alpha of 0.79, 0.86; pre-post intervention, respectively) and comprised of four main constructs: assessing patients' perspectives and opinions (opinion), determining patients' beliefs for history taking and treatment (belief), assessing patients' psychosocial and cultural context (context), and knowing the patients' perspective for providing good health care (quality) (Crosson et al., 2004). However, studies, including ours, have not yielded consistent constructs (Carpenter, Estrada, Medrano, Smith, \& Massie, 2015; De Maesschalck, Willems, De Maeseneer, \& Deveugele, 2010). Among first year medical students, we have shown that the instrument measured two constructs, conceptualized as understanding and utilizing patients perspective and patients' perspective in the patient-physician relationship (Carpenter et al., 2015).

Cultural competency attitudes among medical students early in their training (Carter et al., 2006; Ladson et al., 2006) has not been fully examined. Hence, we examined cultural attitudes of first year medical students and examined the psychometric properties of the scale to better define the constructs it intends to measure.

\section{Methods}

\subsection{Design, Setting, and Participants}

In a cross-sectional study, three consecutive first year medical school classes completed the cultural attitudes survey using a standardized instrument (HBAS); then, we examined internal consistency and construct validity using factor analysis. All first year medical students (matriculating classes of 2011-2013) completed the cultural attitudes survey (described below) within two months of starting medical school their respective matriculating year. The survey was conducted in the context of a required activity as part of the Introduction to Clinical Medicine I (ICM I) curriculum. ICM I focus on the fundamentals of patient centered interviewing and physical diagnosis. Students received no incentives, penalties, or grading for participation. The medical school provided aggregated data on gender, race and ethnicity. The Institutional Review Board at the University of Alabama at Birmingham approved the study on ethical grounds. After reading the informed consent, students marked a checkbox at the beginning of the survey agreeing to participate.

In the first two months of medical school, students are introduced to cultural awareness and cultural sensitivity in various ways. At orientation, students receive information about diversity issues in medicine either through a lecture or role-playing exercise. During the course "Patient, Doctor, and Society," students attend a lecture on cultural competence. As part of ICM I, all students were required to complete web-based training in an independent study fashion. The web-based training consisted of four highly interactive case-based modules emphasizing cross-cultural approaches to care for African-American patients with cardiovascular disease, especially hypertension (Carpenter et al., 2015). The modules available in the public domain (Anonymous, 2015) were designed and implemented after a rigorous formative evaluation process (Crenshaw et al., 2011).

\subsection{Measures}

We assessed cultural attitudes using a standardized instrument, the Health Beliefs Attitudes Survey (HBAS) (Crosson et al., 2004). Students completed the HBAS within two weeks after completion of online modules (described above). The HBAS consists of 15 items, scored on a 6-point Likert scale (1=strongly disagree, $2=$ moderately disagree, $3=$ mildly disagree, $4=$ mildly agree, $5=$ moderately agree, $6=$ strongly agree; higher score indicates more culturally competent attitudes). The HBAS examines attitudes on the relationship of cultural competency to quality healthcare and has been partially validated (excellent internal consistency, Cronbach's alpha $0.79-0.86$; previously reported mean values of 4.4 - 5.4) (Crosson et al., 2004). The survey was not anonymous. 


\subsection{Statistical Approach}

We provide standard descriptive statistics for the overall HBAS score. Following instructions from the original report, the scale was reversed for four questions (questions 3, 5, 7, 15); the four questions were written in the negative to minimize social desirability. The overall HBAS score was the mean of all 15 questions (Crosson et al., 2004). We used the Kruskal-Wallis test to compare overall HBAS scores by matriculating class (data was not normally distributed).

To examine the constructs, we performed factor analysis and grouped the questions based on factors with Eigenvalues greater or equal than one and questions with rotated factors loading greater than 0.3 . We also explored a four-factor solution as prior work has suggested four domains. We conceptualized and assigned a label to the two or four factors by using an iterative approach between the authors (JEC, CAE) based on prior publications. We assessed internal consistency with Cronbach's alpha. We used STATA 11.2 software (StataCorp, 2015) for analyses and defined statistical significance at a $\mathrm{p}$ value $<0.05$.

\section{Results}

The matriculating classes of 2011-2013 consisted of 548 students; $42.2 \%$ of whom were female, $73.0 \%$ were white, $14.6 \%$ were Asian, race/ethnicity were not reported in $8.7 \%$, Table 1 . The HBAS survey was completed by $97.8 \%$ $(536 / 548)$.

Table 1. First Year Medical Student Demographics

\begin{tabular}{lcccc}
\hline & $\begin{array}{c}\text { Class of 2011 } \\
(\mathrm{n}=185)\end{array}$ & $\begin{array}{c}\text { Class of 2012 } \\
(\mathrm{n}=176)\end{array}$ & $\begin{array}{c}\text { Class of 2013 } \\
(\mathrm{n}=187)\end{array}$ & $\begin{array}{c}\text { Total } \\
(\mathrm{n}=548)\end{array}$ \\
\hline Gender & & & & \\
Male & $94(50.8 \%)$ & $106(60.2 \%)$ & $106(56.7 \%)$ & $306(55.8 \%)$ \\
Female & $82(44.3 \%)$ & $70(39.8 \%)$ & $79(42.2 \%)$ & $231(42.2 \%)$ \\
Not Available & $9(4.9 \%)$ & $0(0.0 \%)$ & $2(1.1 \%)$ & $11(2.0 \%)$ \\
& & & & \\
Race/Ethnicity & & & & \\
White & $129(69.7 \%)$ & $135(76.7 \%)$ & $136(72.7 \%)$ & $400(73.0 \%)$ \\
Asian & $29(15.7 \%)$ & $27(15.4 \%)$ & $24(12.8 \%)$ & $80(14.6 \%)$ \\
Black or African American & $7(3.8 \%)$ & $6(3.4 \%)$ & $11(5.9 \%)$ & $24(4.4 \%)$ \\
Hispanic or Latino & $2(1.1 \%)$ & $2(1.1 \%)$ & $6(3.2 \%)$ & $10(1.8 \%)$ \\
American Indian or Alaska Native & $1(0.5 \%)$ & $2(1.1 \%)$ & $3(1.6 \%)$ & $6(1.1 \%)$ \\
Native Hawaiian or Pacific Islander & $1(0.5 \%)$ & $0(0.0 \%)$ & $0(0.0 \%)$ & $1(0.2 \%)$ \\
No Response & $16(8.7 \%)$ & $4(2.3 \%)$ & $7(3.8 \%)$ & $27(4.9 \%)$ \\
\hline
\end{tabular}

\subsection{Cultural Competency Attitudes}

For all matriculating classes, the HBAS median score was 5.3 with $25^{\text {th }}$ percentile [Q1] and $75^{\text {th }}$ percentile [Q3] being 4.9 and 5.7, respectively (in a Likert scale of 1-6). The HBAS score was statistically different among the three matriculating classes, median $5.3(\mathrm{Q} 1,4.8$; Q3, 5.6) for matriculating class of 2011, median 5.5 (Q1, 4.9; Q3, 5.7) for matriculating class of 2012, and median 5.4 (Q1, 5.1; Q3, 5.7) for matriculating class of 2013 (Kruskal-Wallis test, chi-squared $=9.126$ with 2 d.f., $\mathrm{p}=0.01$ )(pairwise comparisons were not performed), Figure.

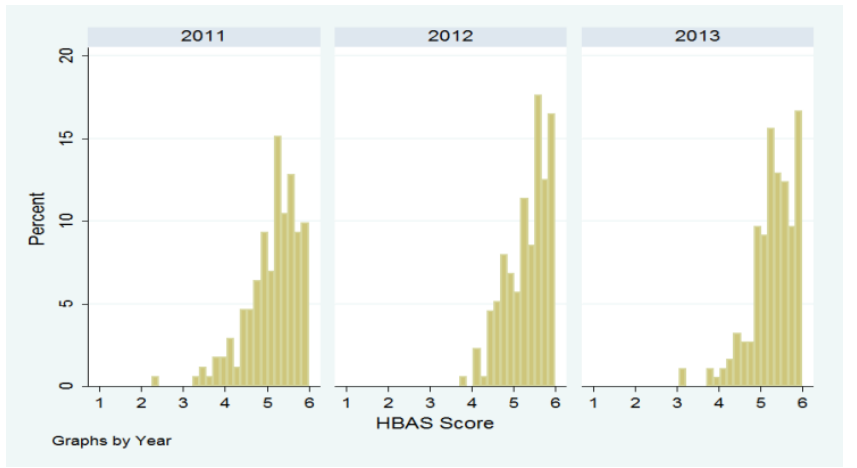

Figure 1. Health Beliefs Attitudes Survey (HBAS) scores by matriculating class.

\subsection{HBAS Instrument-Two-Factor Solution Factor Analysis}

The rotated factor loadings for each question organized by two factors are shown in Table 2. A two-factor solution explained $97 \%$ of the variance with Eigenvalues of 5.6 and 1.2, respectively. By reviewing the wording of the questions for the two factors, we conceptualized Factor 1 as "Understanding the Patient's Cultural and Socio-Economic Background" and Factor 2 as "Building the Professional Relationship and Quality of Care." The Cronbach's alphas 
(internal consistency) were 0.89 and 0.74 , respectively.

Table 2. Health Beliefs Attitudes Survey (HBAS). Factor Analysis

\begin{tabular}{|c|c|c|}
\hline \multirow[t]{2}{*}{ Factors / HBAS Question.* } & \multicolumn{2}{|c|}{ Factor Loading } \\
\hline & $\begin{array}{c}\text { Two } \\
\text { Factors }\end{array}$ & $\begin{array}{c}\text { Four } \\
\text { Factors }\end{array}$ \\
\hline \multicolumn{3}{|l|}{ Factor 1. (Cronbach's alpha $=0.89)$} \\
\hline $\begin{array}{l}\text { Understanding the Patient's Cultural and Socio-Economic Background } \\
\text { Factor A. (Cronbach's alpha }=0.86 \text { ) } \\
\text { Improved Quality of Care through Empathy, Cultural Understanding, and Patient's Perspective }\end{array}$ & & \\
\hline 6 Understanding patients' opinions about their illnesses helps physicians provide better care. & 0.712 & 0.530 \\
\hline 9 A physician should learn about their patients' cultural perspective. & 0.602 & 0.580 \\
\hline 10 Physicians can learn from their patients' perspectives on their illnesses or problems. & 0.680 & 0.513 \\
\hline 12 Physicians should ask about how an illness is impacting a patients' life. & 0.681 & 0.654 \\
\hline 13 Physicians should make empathetic statements about their patients' illnesses or problems. & 0.565 & 0.591 \\
\hline 14 Physicians should ask patients for their feeling about their illnesses or problems. & 0.756 & 0.705 \\
\hline \multicolumn{3}{|l|}{ Factor B. (Cronbach's alpha $=0.79$ ) } \\
\hline \multicolumn{3}{|l|}{ Understanding Patient's Beliefs and Opinions } \\
\hline 1 Physicians should ask patients for their opinions about illnesses or problems. & 0.581 & 0.366 \\
\hline 8 Physicians should ask their patients what they believe is the cause of their problem/illness. & 0.720 & 0.637 \\
\hline 11 Physicians should ask their patients why they think their illness has occurred. & 0.732 & 0.650 \\
\hline \multicolumn{3}{|l|}{ Factor C. (Cronbach's alpha $=0.77)$} \\
\hline \multicolumn{3}{|l|}{ Importance of Patient's Opinion in Diagnosis } \\
\hline 2 It is important to know the patients' points of view for the purpose of diagnosis. & 0.513 & 0.681 \\
\hline $\begin{array}{l}4 \text { Understanding patients' opinions about their illnesses helps physicians reach the correct } \\
\text { diagnosis. }\end{array}$ & 0.499 & 0.698 \\
\hline \multicolumn{3}{|l|}{ Factor 2. (Cronbach's alpha $=0.74)$} \\
\hline \multirow{2}{*}{\multicolumn{3}{|c|}{$\begin{array}{l}\text { Building the Professional Relationship and Quality of Care } \\
\text { Factor D. (Cronbach's alpha }=0.74 \text { ) }\end{array}$}} \\
\hline & & \\
\hline \multicolumn{3}{|l|}{ Building the Professional Relationship and Quality of Care } \\
\hline $\begin{array}{l}3 * \text { Patients may lose confidence in the physician if the physician asks their opinion about } \\
\text { their illness or problem. }\end{array}$ & 0.340 & 0.341 \\
\hline $\begin{array}{l}5^{*} \text { A physician can give excellent care without knowing patients' opinions about their } \\
\text { illnesses or problems. }\end{array}$ & 0.761 & 0.747 \\
\hline $\begin{array}{l}\text { 7* A physician can give excellent health care without knowing a patient's understanding of } \\
\text { his or her illness. }\end{array}$ & 0.779 & 0.772 \\
\hline $\begin{array}{l}\text { 15* Physicians do not need to ask about patients' personal lives or relationships to provide } \\
\text { good health care. }\end{array}$ & 0.567 & 0.563 \\
\hline
\end{tabular}

* Numbers represent survey question number. Following instructions from the original report, the Likert scale reversed for questions 3, 5, 7, and 15 (questions were written in the negative to minimize social desirability).

\subsection{HBAS Instrument - Four-Factor Solution Factor Analysis}

The rotated factor loadings for each question organized by four factors are shown in Table 2. Similarly, we conceptualized Factor A as "Improved Quality of Care through Empathy, Cultural Understanding, and Patient's Perspective," Factor B as "Understanding Patient's Beliefs and Opinions," Factor C as "Importance of Patient's Opinion in Diagnosis," and Factor D as "Building the Professional Relationship and Quality of Care." The Cronbach's alphas (internal consistency) were $0.86,0.79,0.77$, and 0.74 , respectively.

\section{Discussion}

\subsection{Main Study Findings}

In this study, we examined cultural attitudes of first year medical students and examined the internal consistency and construct validity of the scale. First year medical students early in training have high culturally relevant attitudes, as it relates to the medical interview. Although we observed statistical significant differences in scores between the three matriculating classes, the magnitude of the difference ( 0.1 to 0.2 differences between medians) is doubtful of any significance. We also found that the health-beliefs attitudes survey (HBAS) better explores two constructs, rather than the four previously reported in the literature. The factors were understanding the patient background and perspective (11 questions, Cronbach's alpha, 0.89) and building the professional relationship to provide high quality care (4 questions, Cronbach's alpha, 0.74).

\subsection{Cultural Competency Attitudes - Health Belief Assessment Survey}

To our knowledge, three studies have used the HBAS to assess its psychometric properties or measure attitudes in health care (Carpenter et al., 2015; Crosson et al., 2004; De Maesschalck et al., 2010). Crosson et al measured the attitudes among 91 first year medical students before and after two longitudinal courses, described in detail elsewhere, 
at a single medical school (Crosson et al., 2004). The response rate was $65 \%$ before the courses and $86 \%$ after the courses. The authors identified four constructs with modest internal consistency: opinion ( 5 items), belief (4 items), context ( 3 items), and quality (3 items)(Cronbach's alpha, 0.76, 0.67, 0.64, 0.61; respectively). Carpenter et al assessed the attitudes among 180 first year medical students early in their training in a randomized trial of two educational interventions at our institution (95\% response rate) (Carpenter et al., 2015). The authors identified two constructs: understanding and utilizing patients' perspective (9 items) and patients' perspective in the patient-physician relationship (6 items). The Cronbach's alpha internal consistency for the two constructs was good (0.83) to acceptable (0.69); respectively. De Maesschalck (De Maesschalck et al., 2010) surveyed 112 family physicians in Belgium to develop a new instrument to measure attitudes toward ethnic minority patients; assessing the psychometric properties of the HBAS was not within the scope of their study.

Taken together, studies had relatively smaller sample size (Crosson et al., 2004; De Maesschalck et al., 2010) as compared the current study. The two constructs we found in this study with a larger sample - understanding the patient background and perspective and building the professional relationship- are very similar to the two constructs in our prior report with a smaller group of first year medical students (Carpenter et al., 2015).

\subsection{Cultural Competency among Medical Students}

Studies have examined cultural competency among medical students with scales different than the HBAS. Carter et al report the results of a workshop to increase cultural proficiency of 196 third year medical students using an 11-item new scale, the Cultural Attitudes and Belief Scale (CABS; 5-point Likert scale)(Carter et al., 2006). In this study, the authors examined the effectiveness of an interactive workshop to improve attitudes, beliefs, and communication skills during the family medicine clerkship (clinical rotation as part of medical school curriculum). The three-hour cultural competency workshop at the start of the clinical rotation included lectures, and case-scenarios showcasing communication between patients and physicians (both effective and ineffective). The post-workshop mean scores ranged from 2.7 to 4.2 .

a) The two items with mean values less than 3 were 'I am an unbiased individual' and 'At least initially, cultural competency is more important than technical competency.'

b) The two items with mean values over 4 were 'A particular lifestyle or culture is irrelevant when it comes to good medical care' and 'I believe that race, religion and culture shold play little role or no part in the assessment and treatment of patients.'

c) In summary, these four items illustrate that one extreme of the scale does not mean better or worse cultural competency attitudes; hence, we cannot compare with the results in our study.

Ladson et al describe domains of cultural competency of 95 first and second year students at historically black medical school using a 90-item previously described scale, the Clinical Culture Competency Questionnaire (CCCQ; 5-point Likert scale)(Ladson et al., 2006). The CCCQ examine 5 domains of cultural competency: knowledge, skills, encounters/situations, attitudes, education, and training/impact.

d) The 18 items included in the attitude subscore had a mean value of 3.5 (converted to a 5-point Likert). A higher value in the score suggests more open attitudes towards cultural competency.

e) The value in this study of first and second year medical students is lower than the value we obtained; however, the questions were different from the ones used in our study.

\subsection{Limitations}

First, the study was conducted at a single academic institution. However, as a state-supported medical school, incoming students are from a wide variety of social and rural backgrounds from the Southeast United States. Second, testing was done early in the students' training and shortly after taking courses of cultural competency; thus, the high survey scores may have over estimated their attitudes, represent social desirability, or a ceiling effect. Finally, the study was not designed to assess skills or knowledge and we did not collect attitudes later in training.

\subsection{Conclusions}

Cultural competency in medical education has emerged as providers require the skills to care for a diverse patient population. Early in training, medical students have high culturally-relevant attitudes as it relates to the medical interview. Based on our study, the HBAS instrument captures two main constructs, understanding the patients' background and perspective and building the professional relationship.

\section{Acknowledgements}

This study was funded by the National Heart, Lung, and Blood Institute (K07 HL081373-01) to Dr. Estrada. The opinions expressed in this article are those of the authors alone and do not reflect the views of the Department of 
Veterans Affairs. The authors report no conflict of interest.

\section{Conflict of Interest}

The authors have no conflict of interest to report. All authors have read this manuscript and given their permission for it to be published.

\section{References}

Anonymous. (2015). Cultural Competence Online for Medical Practice. Retrieved from http://www.c-comp.org.

Beach, M. C., Price, E. G., Gary, T. L., Robinson, K. A., Gozu, A., Palacio, A., \& Cooper, L. A. (2005). Cultural competence: a systematic review of health care provider educational interventions. Med Care, 43(4), 356-373. http://dx.doi.org/10.1097/01.mlr.0000156861.58905.96

Betancourt, J. R., \& Green, A. R. (2010). Commentary: linking cultural competence training to improved health outcomes: perspectives from the field. Acad Med, 85(4), 583-585. http://dx.doi.org/10.1097/ACM.0b013e3181d2b2f3

Carpenter, R., Estrada, C. A., Medrano, M., Smith, A., \& Massie, F. S. Jr. (2015). A web-based cultural competency training for medical students: a randomized trial. Am J Med Sci, 349(5), 442-446. http://dx.doi.org/10.1097/MAJ.0000000000000351

Carrillo, J. E., Green, A. R., \& Betancourt, J. R. (1999). Cross-cultural primary care: a patient-based approach. Ann Intern Med, 130(10), 829-834. http://dx.doi.org/10.7326/0003-4819-130-10-199905180-00017

Carter, M. M., Lewis, E. L., Sbrocco, T., Tanenbaum, R., Oswald, J. C., Sykora, W., \& Hill, L. D. (2006). Cultural competency training for third-year clerkship students: effects of an interactive workshop on student attitudes. $J$ Natl Med Assoc, 98(11), 1772-1778.

Crenshaw, K., Shewchuk, R. M., Qu, H., Staton, L. J., Bigby, J. A., Houston, T. K., \& Estrada, C. A. (2011). What should we include in a cultural competence curriculum? An emerging formative evaluation process to foster curriculum development. Acad Med, 86(3), 333-341. http://dx.doi.org/10.1097/ACM.0b013e3182087314

Crosson, J. C., Deng, W., Brazeau, C., Boyd, L., \& Soto-Greene, M. (2004). Evaluating the effect of cultural competency training on medical student attitudes. Fam Med, 36(3), 199-203.

De Maesschalck, S., Willems, S., De Maeseneer, J., \& Deveugele, M. (2010). Development and Validation of EMP-3: An Instrument to Measure Physician's Attitudes Toward Ethnic Minority Patients. Fam Med, 42(4), 262-267.

Dobbie, A. E., Medrano, M., Tysinger, J., \& Olney, C. (2003). The BELIEF Instrument: a preclinical teaching tool to elicit patients' health beliefs. Fam Med, 35(5), 316-319.

Harris-Haywood, S., Goode, T., Gao, Y., Smith, K., Bronheim, S., Flocke, S. A., \& Zyzanski, S. (2014). Psychometric evaluation of a cultural competency assessment instrument for health professionals. Medical Care, 52(2), e7-e15. http://dx.doi.org/10.1097/MLR.0b013e31824df149

Horvat, L., Horey, D., Romios, P., \& Kis-Rigo, J. (2014). Cultural competence education for health professionals. Cochrane Database Syst Rev, 5, Art. No.: CD009405. http://dx.doi.org/10.1002/14651858.CD009405.pub2

Kardong-Edgren, S., \& Campinha-Bacote, J. (2008). Cultural competency of graduating US Bachelor of Science nursing students. Contemp Nurse, 28(1-2), 37-44. http://dx.doi.org/10.5172/conu.673.28.1-2.37

Ladson, G. M., Lin, J. M., Flores, A., \& Magrane, D. (2006). An assessment of cultural competence of first- and second-year medical students at a historically diverse medical school. Am J Obstet Gynecol, 195(5), 1457-1462. http://dx.doi.org/10.1016/j.ajog.2006.06.051

LCME. (2015). Liaison Committee on Medical Education. Functions and Structure of a Medical School. http://www.lcme.org/publications.htm.

Lie, D. A., Lee-Rey, E., Gomez, A., Bereknyei, S., \& Braddock, C. H., 3rd. (2011). Does cultural competency training of health professionals improve patient outcomes? A systematic review and proposed algorithm for future research. J Gen Intern Med, 26(3), 317-325. http://dx.doi.org/10.1007/s11606-010-1529-0

Loftin, C., Hartin, V., Branson, M., \& Reyes, H. (2013). Measures of cultural competence in nurses: an integrative review. ScientificWorldJournal, 2013, 289101. http://dx.doi.org/10.1155/2013/289101

Nazar, M., Kendall, K., Day, L., \& Nazar, H. (2014). Decolonising medical curricula through diversity education: Lessons from students. Med Teach, 1-9. http://dx.doi.org/10.3109/0142159x.2014.947938 
Pena Dolhun, E., Munoz, C., \& Grumbach, K. (2003). Cross-cultural Education in U.S. Medical Schools: Development of an Assessment Tool. Acad Med, 78(6), 615-622. http://dx.doi.org/10.1097/00001888-200306000-00012

Price, E. G., Beach, M. C., Gary, T. L., Robinson, K. A., Gozu, A., Palacio, A., \& Cooper, L. A. (2005). A systematic review of the methodological rigor of studies evaluating cultural competence training of health professionals. Acad Med, 80(6), 578-586. http://dx.doi.org/10.1097/01.mlr.0000156861.58905.96

Stata Corp. (2015). STATA 11.2 (College Station, Texas, USA.

Truong, M., Paradies, Y., \& Priest, N. (2014). Interventions to improve cultural competency in healthcare: a systematic review of reviews. BMC Health Serv Res, 14, 99. http://dx.doi.org/10.1186/1472-6963-14-99

This work is licensed under a Creative Commons Attribution 3.0 License. 